\title{
Chapter 15 \\ Resilience: From Practice to Theory and Back Again
}

\author{
Carl Macrae and Siri Wiig
}

\begin{abstract}
This book offers a purposefully broad exploration of resilience: it presents a variety of diverse perspectives in a range of practical contexts across various scales of system from a range of disciplinary positions. One of the core organising principles of this book is a concern with understanding how ideas of resilience can be translated into practice, and how practices of resilience can in turn be theorised and explainedirrespective of whether those practices are conducted at the 'street-level' by frontline actors or in the committee rooms of policymakers. To do this, the book explores empirical, methodological and theoretical challenges in analysing resilience, defining resilience, organising resilience, building resilience, leading resilience and regulating resilience - to name just a few of the activities that provide the focus of concern in these chapters. In this chapter, we provide a brief and necessarily partial survey of the varieties and commonalities of resilience that have emerged throughout the book, and then explore how — and why — we might move towards an integrated theoretical framework of resilience.
\end{abstract}

Keywords Theory $\cdot$ Integrative framework $\cdot$ Resilience studies $\cdot$ Systems improvement

\subsection{Varieties of Resilience: A Tour of the Landscape}

Throughout this book one of the most apparent, and perhaps inevitable, characteristics of resilience is its variety. From the basic definitions and objectives of resilience to the ways in which it is operationalised in different settings, it is clear that one of the strengths of the concept of resilience is its ability to accommodate a broad set

\footnotetext{
C. Macrae ( $\square)$

University of Nottingham, Nottingham, UK

e-mail: carlmacrae@mac.com
}

\section{S. Wiig}

Faculty of Health Sciences, SHARE-Center for Resilience in Healthcare,

University of Stavanger, Stavanger, Norway 
of phenomena in many settings and at different levels and scales of activity. This allows disparate and diverse practices to be analysed through the same conceptual lens - from the second-by-second work of air traffic controllers (Woltjer, Chap. 13) to the long-term organisation of global humanitarian efforts (Kimber, Chap. 11; Tjoflat and Hansen, Chap. 12). However, this breadth can also be a source of weakness, and points to the risk of theoretical over-reach: if a concept attempts to explain everything it can end up explaining nothing. Nonetheless, across the book a set of core ideas and principles can be seen emerging amongst the varied perspectives. For instance, definitions of what resilience is clearly vary, but there is general agreement on core characteristics: primarily the ability of an entity-individuals, communities, organisational units or larger systems - to return to some 'normal' condition or state of functioning after an event that disrupts its state; or to adapt to a new normal state, where system functioning is reorganised or enhanced in some way in response to the disruption. Basic definitional issues such as this are not simply of theoretical concern but have deeply practical consequences. As Kimber (Chap. 11) argues, a vague definition of resilience presents challenges for creating and managing resilience in operational activities — while also acting as a valuable 'boundary object' that can bring together different communities around a (loosely defined) shared goal. In practice, as in theory, the emerging field of resilience studies needs to engage in an ongoing negotiation to ensure theories of resilience can both support the benefits of coordinating diverse communities and perspectives, while also providing the degree of detail and specificity needed to operationalise and use those theories in different domains.

Another variety of resilience explored throughout the book concerns the level of analysis and scale of activity that provides the focus for understanding resilience. Resilience is inherently a systems-oriented concept: it can be applied at different levels of a system, from the level of individual cognition to entire societies and beyond; and it can be used to examine the complex interrelations and interconnections between different levels and scales of these systems. Again, this breadth would appear to be both a source of strength and weakness. The moment-by-moment activities and cognitive processes of individuals can seem far removed from the large-scale adaptations and reorganisations that unfold across entire industries, and studying these interrelationships at vastly different scales of time and space presents considerable methodological challenges. As Le Coze describes (Chap. 2), these challenges include long time scales, complex networks of stakeholders, distributed research sites, and a large quantity and wide variety of data. Nonetheless, the work presented in this book indicates that, to better grapple with key issues in theorising and operationalising resilience, we need to be much more attentive to the levels or 'scales' of activity at which resilience unfolds, along with the different forms, functions and characteristics of resilience at these different scales of activity. Kyriakidis and Dang (Chap.6) and Woltjer (Chap. 13) use the domains of critical infrastructure and air traffic control to explore how resilience depends on interactions and adaptations that interconnect across different system levels, and analyse how people at all levels of a system can contribute (or not) to resilience by adapting performance to local conditions. For example, Kyriakidis and Dang (Chap.6) argue that frontline personnel are involved in short-term adaptations when monitoring and responding to service deviation, but 
it requires higher-order managerial and organisational responses to effectively learn from an organizations' past experience and anticipate future threats-processes they conceptualise as a 'resilience capability loop' that organises the activities of anticipation, monitoring, responding, and learning. Some of these ideas are echoed in Macrae's (Chap. 3) framework of resilience at different scales of sociotechnical activity, from situated and immediate responses that unfold rapidly, to structural adaptations that involve longer processes of reorganisation, to long-term systemic reconfigurations involving system-wide reform.

One of the challenges of understanding resilience as a truly system-level phenomenon across different scales of activity is exemplified by the work of Berg and Aase (Chap. 10) in the context of healthcare: research on resilience has, to date, mostly focused on those working at the 'front line' or 'sharp end' of organisational practice. And, in the domain of healthcare, little systematic empirical attention has been paid to the higher-level systems activities at the macro level, encompassing the role of national bodies and regulatory agencies. This knowledge gap echoes experience in other fields, where our understanding of resilience mechanisms within and across regulatory actors, or the networks that span entire industries, is lacking (eg Woltjer, Chap. 13). For example, what forms does ongoing adaptation take within regulators, in response to challenge and feedback from regulated organizations, the public, or stakeholders [1]? More broadly, understanding the roles of different stakeholders in the active 'co-creation' of resilience is emerging as a key issue and focus for future empirical work, particularly in the contexts of healthcare, disaster planning and recovery, and international operations. Tjoflåt and Hansen's (Chap. 12) experience with humanitarian programs in protracted conflicts indicate how working in partnership with people and local communities is essential in building resilience. Similarly, Baram (Chap. 5) describes how acknowledging and valuing inter-organizational dependencies is central to building resilience in public water supply systems. Locally-based organizations and networks of other stakeholders can provide public support and contribute to both preventative and recovery efforts in critical infrastructure. One of the practical implications of this is explored by Chambers and Storm (Chap. 14). Understanding the co-creation of resilience requires more systematic and routine stakeholder analysis — both in research and practice - to identify and maximise mechanisms of co-creating resilience across levels of socio-technical systems, and also to assess potential 'risk makers' who may destabilise those efforts of co-creation.

Ultimately, perhaps one of the most practical issues explored by the book is: to what extent is it possible to design and implement resilience-as an individual, an organisation, a leader, or across an entire system? And, what strategies or methods might support practitioners in this? Leadership is particularly important in creating resilient organisations, and some of the key leadership challenges are described by Grote (Chap. 8) - particularly the ongoing and delicate balance required to match stability and flexibility demands in organizations and create environments for resilient performance. Leaders themselves, just like their organisations, need to be able to function in different modes of operation, train their adaptive capabilities and manage rapid mode shifts in response to changing conditions. Sophisticated organisa- 
tional structures and processes are also needed to support resilience. Pettersen Gould (Chap.7) examines how airlines adopt meso-level strategies of resilience that support the collection of organisation-wide operational data, operational planning and design, and coordinated action to adapt and improve operational activities. By promoting continuous monitoring, analysis, and attentiveness to possible adaptions in pilots' landing approaches, airline managers enact systems that support 'precursor resilience' - rapidly addressing disruptions and fluctuations to maintain safe performance. At the level of specific organisational interventions, both Anderson et al. (Chap.4) and Reiman and Viitanen (Chap.9) provide rich demonstrations of how particular improvement methods, checklists and related sociotechnical tools can be implemented to support adaptive processes of improvement and safety management. Drawing on the contrasting settings of healthcare, nuclear power and beyond, Anderson et al., Reiman and Viitanen document ways in which a variety of practical tools can be integrated into (and evaluated as part of) more strategic programmes of resilience engineering.

\subsection{Towards an Integrative Framework of Resilience}

Taken together, these chapters sketch out a rich variety of applications, complications and opportunities for the field of resilience, in both theory and practice. This variety points to the wide landscape of resilience that is only beginning to be systematically explored. This variety also presents clear challenges for the future of this developing field. These include the challenges of communicating across disciplinary perspectives, working at different scales of system and levels of analysis, integrating insights across different applied domains and practical settings, and ultimately connecting theory and practice in ways that are productive for both. It is also striking that many of the challenges of developing this field echo those of enacting resilience itselfsuch as engaging diverse stakeholders, adaptively shifting perspectives and modes of operation depending on context, and updating and integrating models to account for local variation. Addressing these challenges points to the potential value of a broad, integrative framework to help support and coordinate cross-domain, international, multi-level and interdisciplinary work on understanding and operationalizing resilience in complex sociotechnical systems. Resilience is an expansive field of research encompassing many different domains and issues-indeed, that is one of its defining strengths. Clearly no single theory could be expected to properly capture and explain all the relevant factors, concepts, relations, and logics that would constitute a fully developed and all-encompassing theory [2]. However, mapping out the general contours of a broad and expansive framework may act as a useful coordinating device for a range of future work, and help to highlight future questions and shape future objectives in both the research community and for practitioners in high-risk industries.

Building on the work in this book, one useful place to start in developing such a framework would be to identify the underlying and most basic commitments that 
might underpin an integrative framework of resilience. Perhaps the most foundational principle here is that all organised human and technical activity is constituted by some degree of inherent fluctuation and variation; and that resilience represents the active and effortful application of different sociotechnical resources (skills, knowledge, relationships, equipment, values, creativity, etc) to handle those moments of disruption that threaten current goals. This basic principle appears to rest on four core assumptions that guide much work in this field, and this book. First, ideas of resilience focus on, and have a deep concern with, acting in the world; a key focus of resilience is always some sort of ongoing practical, situated activity in a complex, socially organized 'real-world' setting. A second assumption is that mechanisms of resilience broadly involve the deployment of certain skills, capabilities or resources to handle a particular challenge or stress. A third assumption is that processes of resilience are inherently dynamic, involving change and adaptation in complex multifactorial and multi-level systems. A forth assumption is that resilience, in its broadest conception, is fundamentally a dualistic concept: it is about conserving and maintaining some set of functions or goals, and achieving this through (or despite) changes, adaptations and reformulations of ongoing activities and performances.

While this list is by no means exhaustive, this core principle and these four assumptions appear to exemplify commonalities across many ideas and ideals of resilience, and appear to be woven through many of the underlying commitments that are explored and operationalised throughout this book. If this is correct, then these are the types of basic commitments that can help form the basis of a future integrative framework of resilience. They also point to some of the more granular questions that can frame an integrated and programmatic inquiry into the nature of resilience, and that help to transcend any particular disciplinary traditions or domains of application. These questions include:

- What are the core phenomena of resilience? Where and in what ways does resilience unfold in a sociotechnical system, and what is the core focus for study and for management?

- What is the context of resilience? Which sociotechnical factors, processes and elements are central to take into account when explaining and engineering resilience, and which are merely peripheral?

- What are the types of sociotechnical resources that are drawn on to support and enact resilience? How are these drawn on, applied, used, created and renewed? What counts as a resource, to whom, in what circumstances and for what purposes?

- What are the processes through which resilience unfolds? What are the sociotechnical mechanisms that support ongoing adaptation and adjustment? And how are these processes organised, designed and managed?

Many of the explorations in this book, and elsewhere in the literature, offer useful perspectives on these questions. Indeed, any discussion of resilience or effort to operationalize resilience must be based on answers to these questions. However, these answers can often be implicitly assumed rather than explicitly defined. These questions therefore represent another jumping-off point for developing an integrative framework for resilience, helping to define its shape and content. To develop this 


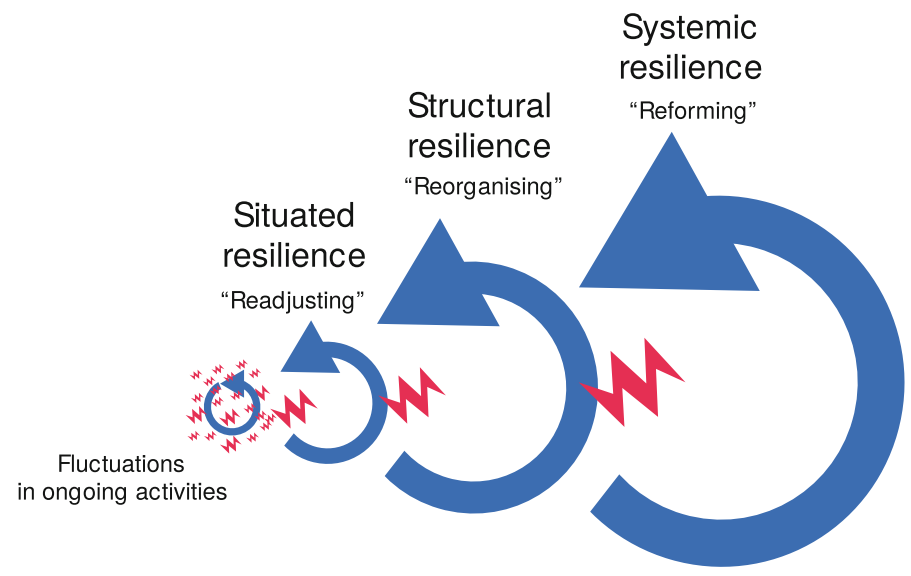

Disruptions to ongoing activities

Fig. 15.1 Resilience at three scales of sociotechnical activity

further, it is useful to revisit the analysis presented by Macrae in Chap. 3 that seeks to characterise resilience at three different scales of sociotechnical activity: situated, structural and systemic. The core assumption is that all organisational activity is inherently variable, and that at some point, those variations can become disruptions to ongoing activities and require the situated application of existing sociotechnical resources to respond, readjust and recover ongoing activities. Sometimes, existing resources may not be adequate to address the scale of disruption at hand, and so structural adaptations may be required, to reorganise existing sociotechnical resources and associated situated practices. On occasion, the arrangements for enacting structural adaptations may not be adequate to address the scale of disruption that has emerged, and so systemic adaptations may be necessary, to reform the systems, processes and structures through which sociotechnical resources and situated practices are designed and produced (Fig. 15.1).

This framework seeks to move beyond definitions of resilience that are tied to particular 'levels' of system structure, or 'types' of resilient capability. Instead, the focus is primarily on providing a general framework and initial language for explaining the processes of resilience at different scales of time and space in complex sociotechnical systems. For instance, activities in what are traditionally viewed as 'macro' level settings (such as national regulatory agencies or supranational bodies) may still represent processes of situated resilience: small scale adaptations to disruptions by applying available and pre-existing sociotechnical resources. Conceptualising resilience in terms of situated practices and sociotechnical resources can help distinguish between activities of resilience that are fundamentally systemic - that involve deeply reforming the foundational elements of how a sociotechnical system is organized and constituted-as opposed to activities that are simply widespread 
but involve no change to the fundamental model of the system, such as lots of people updating a simple local protocol in many different settings across an industry.

Framing resilience in this way provides one example of how an integrative framework might build on the core principles, assumptions and questions outlined above, to move beyond categories and concepts that can sometimes inadvertently maintain disciplinary boundaries or introduce artificial silos into the analysis and explanation of resilient systems. For example, this framing of situated, structural and systemic resilience can provide a framework for focusing attention on the critical questions of how, when and why fluctuations become disruptions, and how disruptions expand in scale to provoke larger moments of resilience that increasingly enroll greater numbers of stakeholders across a system and ultimately challenge, reorganize and reform core elements of that system. To take one practical example from healthcare: media reports and public pressure relating to the handling of a harmful adverse event can act as a significant disruption for a regulatory body, prompting a rapid response to review and reopen an investigation using existing organisational resources and models (situated resilience); which in turn can provoke the design and reorganization of new processes for involving next-of-kin and outside experts in inquiries (structural resilience); and ultimately can lead to system-wide recommendations for reforming the underling mechanisms of collaboration between regulatory bodies involved in assessing compliance with core regulatory requirements (systemic resilience) [3]. This example illustrates how an integrative framework for resilience should be flexible enough to accommodate granular details related to a single organization and event, whilst also providing a language to explain the 'scaling up' of resilience across entire systems and over long time periods. An integrative framework should also accommodate the 'positive', as well as the 'negative', aspects of resilience: the processes of improvement, adaptation and innovation as much as the management of the adverse impacts and crises that are often viewed as the prime triggers of resilience. For instance, in the safety sciences the language of 'disruption' may seem inherently skewed towards the dark side of organisational life and suggestive of harmful or adverse events. However, when viewed through the lens of a framework that is as much focused on the 'positive' processes of adaptation, change and renewal, then 'disruption' can take on a broader meaning. The situations that provoke resilience might always be defined as 'disruptive' to an existing way of doing things or to established cultural assumptions - but that disruption might be in the form of a potentially harmful event, or in the form of the invention of a new and innovative technology. Both of these represent a disruption to an existing world order. An integrative framework of resilience should be able to fully accommodate, and explain, both.

\subsection{Resilience: Between Theory and Practice}

Resilience is a powerful and engaging idea because of its breadth and scope. Likewise, its breadth and scope leave the field of resilience studies open to increasing fragmentation and polarization. Accordingly, it seems important that the field moves 
towards debates that surface the underlying assumptions, principles, commitments and questions that support cross-pollination of ideas, insights and practices. Developing an integrated framework that can accommodate a wide range of concepts, strategies and models of resilience, and offers a way of connecting them and linking them across levels of activity, seems an important part of this journey. In the words of Kurt Lewin, there is nothing quite so practical as a good theory [4]. A good theory should be able to explain, predict, and delight [5]. And theories should be practical things that help us act in, and on, the world. Due to the many varieties of resilience, a single, simple and general theory of resilience is not a realistic objective. But this book indicates that the field would benefit from engaging in a thorough, expansive and ongoing process of theorizing [6] that continues to define and address the many cultural, symbolic, organisational, sociotechnical and practical aspects of resilience that operate across myriad scales of time and space.

\section{References}

1. S.H. Berg, K. Akerjordet, M. Ekstedt, K. Aase, Methodological strategies in resilient health care studies: An integrative review (2018). Resubmitted to Safety Science, referred to with permission from authors

2. D.A. Whetten, What constitutes a theoretical contribution? Acad. Manag. Rev. 14(4), 490-495 (1989)

3. S. Wiig, K. Aase, M. Bourrier, O. Rise, Transparency in health care - displaying adverse events to the public, in Risk Communication for the Future, ed. by M. Bourrier, C. Bieder (Springer, Berlin, 2018), pp. 111-125

4. A.H.V. de Ven, Nothing is quite so practical as a good theory. Acad. Manag. Rev. 14(4), 486-489 (1989)

5. R.I. Sutton, B.M. Staw, What theory is not. Adm. Sci. Q. 40(3), 371-384 (1995)

6. K.E. Weick, What theory is not, theorizing is. Adm. Sci. Q. 40(3), 385-390 (1995)

Open Access This chapter is licensed under the terms of the Creative Commons Attribution 4.0 International License (http://creativecommons.org/licenses/by/4.0/), which permits use, sharing, adaptation, distribution and reproduction in any medium or format, as long as you give appropriate credit to the original author(s) and the source, provide a link to the Creative Commons license and indicate if changes were made.

The images or other third party material in this chapter are included in the chapter's Creative Commons license, unless indicated otherwise in a credit line to the material. If material is not included in the chapter's Creative Commons license and your intended use is not permitted by statutory regulation or exceeds the permitted use, you will need to obtain permission directly from the copyright holder.

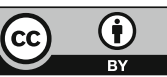

